\title{
KLF8 promotes tumorigenesis, invasion and metastasis of colorectal cancer cells by transcriptional activation of FHL2
}

\author{
Qingqing Yan ${ }^{1}$, Wenjing Zhang ${ }^{1}$, Yao $W_{u^{1}}$, Meiyan $\mathbf{W u}^{1}$, Mengnan Zhang ${ }^{1}$, \\ Xinpeng Shi ${ }^{1}$, Jinjun Zhao ${ }^{2}$, Qingzhen Nan ${ }^{1}$, Ye Chen ${ }^{1}$, Long Wang ${ }^{3}$, Tianming \\ Cheng ${ }^{1}$, Jiachu Li ${ }^{4}$, Yang Bai ${ }^{1}$, Side Liu ${ }^{1}$, Jide Wang ${ }^{1}$ \\ ${ }^{1}$ Guangdong Provincial Key Laboratory of Gastroenterology, Department of Gastroenterology, Nanfang Hospital, Southern \\ Medical University, Guangzhou, China \\ ${ }^{2}$ Department of Rheumatism, Nanfang Hospital, Southern Medical University, Guangzhou, China \\ ${ }^{3}$ Division of Vascular Interventional Radiology, Third Affiliated Hospital, Sun Yat-sen University, Guangzhou, China \\ ${ }^{4}$ Department of Oncology, The First Affiliated Hospital of Chongqing Medical University, China
}

Correspondence to:

Jide Wang, e-mail: jidewang55@163.com

Side Liu, e-mail: liuside@163.com

Keywords: KLF8, EMT, FHL2, metastasis, colorectal cancer

Abbreviations: EMT, epithelial-to-mesenchymal transition; FHL2, four-and-a-half LIM-only protein 2; RLU, relative luciferase unit; ATCC, american type culture collection; ChIP, chromatin immunoprecipitation

Received: December 10,2014 Accepted: July 03, $2015 \quad$ Published: July 15, 2015

\section{ABSTRACT}

The transcription factor Krüppel-like factor (KLF)8 plays an important role in the formation of several human tumors, including colorectal cancer. We recently identified four-and-a-half LIM protein 2 (FHL2) as a critical inducer of the epithelialto-mesenchymal transition (EMT) and invasion. However, the molecular mechanism by which KLF8 affects FHL2-mediated tumor proliferation, EMT and metastasis remains unknown. Here, we showed that KLF8 overexpression promoted EMT and metastatic phenotypes. KLF8 expression was stimulated by transforming growth factor (TGF)- $\beta 1$. Moreover, KLF8 acted as a potential EMT inducer by stimulating vimentin expression and inducing a loss of E-cadherin in stable KLF8-transfected cells. KLF8 overexpression induced a strong increase in FHL2 expression, and a positive correlation between the expression patterns of KLF8 and FHL2 was observed in CRC cells. Promoter reporter and chromatin immunoprecipitation (ChIP) assays demonstrated that KLF8 directly bound to and activated the human FHL2 gene promoter. However, siRNA-mediated repression of $F H L 2$ in KLF8-overexpressing cells reversed the EMT and the proliferative and metastatic phenotypes. In vivo, KLF8 promoted FHL2-mediated proliferation and metastasis via orthotopic implantation. Taken together, this work identified KLF8induced FHL2 activation as a novel and critical signaling mechanism underlying human breast/colorectal cancer invasion and metastasis.

\section{INTRODUCTION}

Specificity protein 1 (Sp1) and other Sp and Krüppellike factor (KLF) proteins are members of a family of transcription factors [1-2]. Sp1-Sp9 proteins regulate expression of multiple genes in normal tissues and tumours $[1,3]$. Other proteins KLFs are essential DNA-binding transcriptional regulators with diverse functions in various cellular processes, including differentiation [4], proliferation [5], inflammation [6], migration [7], and pluripotency [8].
Recent progress has highlighted the significance of KLFs in tumor progression. For example, KLF family proteins have been shown to have either a tumor-promoting (KLF4 [2, 7], KLF6 [9], KLF9 [10], KLF10 [11], KLF11 [12], and KLF17 [13]) or a tumor-suppressing (KLF5 [14], KLF12 [15]) role by intervening in cell signaling associated with proliferation and/or suppression.

KLF8 [16] is a member of the KLF transcription factor family and binds to a DNA consensus sequence (GT-box [17]: 5'-CACCC-3') to regulate transcription. 
KLF8 is ubiquitously expressed in proliferating and regenerating mammalian cells and is highly expressed in various types of human malignancies $[18,19]$. Recent studies have suggested that KLF8 plays an important role in gastric cell development and progression [16]. For example, the downregulation of KLF8 expression inhibited hepatocellular carcinoma cell growth, migration, and invasion by decreasing cyclin D1, Cdk, matrix metalloproteinase (MMP)-2 and MMP-9 expression [17]. In addition, KLF8 induced tumor cell epithelialto-mesenchymal transition (EMT) and maintained the invasive potential of cancer, which appeared to play a crucial role in the metastatic progression of human carcinomas [16].

Four-and-a-half LIM-only protein 2 (FHL2) [20], also known as downregulated in rhabdomyosarcoma LIM (DRAL) domain protein, is a member of the FHL family and comprises four LIM domains and one N-terminal half LIM domain. The LIM domains are double zinc finger motifs that play multiple roles in protein-protein interactions such as functional modifiers and adaptors [21, 22]. FHL2 is a LIM-only protein that participates in cell transcription and signal transduction. It functions as a coactivator of several transcription factors, such as $\beta$-catenin [23], AP-1 [24], CREB [25], and others [26]. We and others have implicated FHL2 as an oncogenic factor in colorectal cancer (CRC) [21], squamous cell carcinoma [27], melanoma [28], glioblastoma [29] and prostate cancer [30]. The over-expression of FHL2 in CRC cells is essential to maintain their malignant phenotype and might be a mediator or a trigger of EMT in CRC cells [23, 31]. We identified the positive correlation between $\mathrm{Sp} 1$ and FHL2 in expressing pattern of GI cancers [3]. However, the mechanism by which KLF8 regulates FHL2 expression through transcriptional activation to promote cell growth, EMT, invasion and metastasis has not been investigated.

In this study, we found that KLF8 overexpression promotes EMT and invasive and metastatic phenotypes. KLF8 regulated FHL2 expression through the GT-box located in the FHL2 proximal promoter. FHL2 short interfering RNA (siRNA) reversed these effects in cells that overexpressed KLF8. These results indicated that tumor progression and metastasis were promoted by a key signaling pathway involving KLF8-mediated regulation of FHL2. These findings further uncovered the role of KLF8 in CRC invasion and metastasis.

\section{RESULTS}

\section{KLF8 upregulation promotes epithelial- mesenchymal transition (EMT)}

EMT is the key process that drives cancer metastasis [7, 13, 16, 23, 31-34]. The loss of E-cadherin expression and gain of vimentin expression are considered to be the most important molecular markers of EMT. Our previous reports demonstrated that KLF8 was overexpressed in CRC (Shi X, submitted). Here, we demonstrated that KLF8 overexpression promotes EMT in CRC in LoVo cells. KLF8 overexpression was confirmed by western blot analysis (Figure 1A). EMT induction was demonstrated by a shift in the expression of epithelial markers (E-cadherin) to mesenchymal markers (vimentin and N-cadherin; Figure 1A).

We also demonstrated increased migration and invasion. As assessed by a wound-healing assay, in which a gap is formed in a cell monolayer, KLF8 overexpression markedly accelerated cell migration (Figure 1B). Similarly, KLF8 overexpression increased LoVo cell invasion by $415.0 \%$ compared with the control cells (Figure 1C). Our results demonstrated that KLF8 overexpression induced EMT and promoted migration and metastasis.

To identify the role of KLF8 in EMT, we first evaluated its response to the most potent EMT inducer, TGF- $\beta 1$. We showed that TGF- $\beta 1$ induced KLF8 in a dose-dependent manner, as determined by western blotting. TGF- $\beta 1$ markedly induced the expression of the EMT marker vimentin (Figure 1D). Blocking TGF- $\beta 1$ with its neutralizing antibody significantly suppressed endogenous KLF8 expression and TGF- $\beta$-induced KLF8 expression in LoVo cells (Figure 1E).

However, pre-transfecting cells with KLF8 siRNA not only inhibited KLF8 expression but also suppressed endogenous and TGF- $\beta$-induced vimentin expression (Figure 2F). These results implicated KLF8 as a responsive factor of EMT induction.

\section{Positive correlation between KLF8 and FHL2 expression in CRC}

To elucidate the correlation between KLF8 and FHL2 in cancer cells, we first checked the expression of these proteins in CRC cell lines by western blot. As shown in Figure 2A, KLF8 was expressed at a relatively high level in SW480, SW1116, Caco2 and SW620 cells and at a relatively low level in HT29, DLD1 and LoVo cells. The FHL2 expression pattern was very similar to that of KLF8 in the above 7 cell lines, except for SW480 cells. Secondly, we investigated the cellular distribution of the two proteins. A two-color immunofluorescence assay showed that the endogenous KLF8 and FHL2 proteins localized to both the nucleus and the cytoplasm of LoVo cells and SW620 cells (Figure 2B). A merged signal indicates the co-localization of the two proteins.

To validate our findings in vivo, we detected KLF8 and FHL2 expression in 13 pairs of adjacent normal colon mucosal tissues and cancer tissues by immunohistochemistry (IHC), as described above. We showed that both proteins were highly expressed by cancer cells, whereas their expressions in normal tissues were absent or extremely low (Figure 2C). Observations using serial sections showed that KLF8 and FHL2 were 


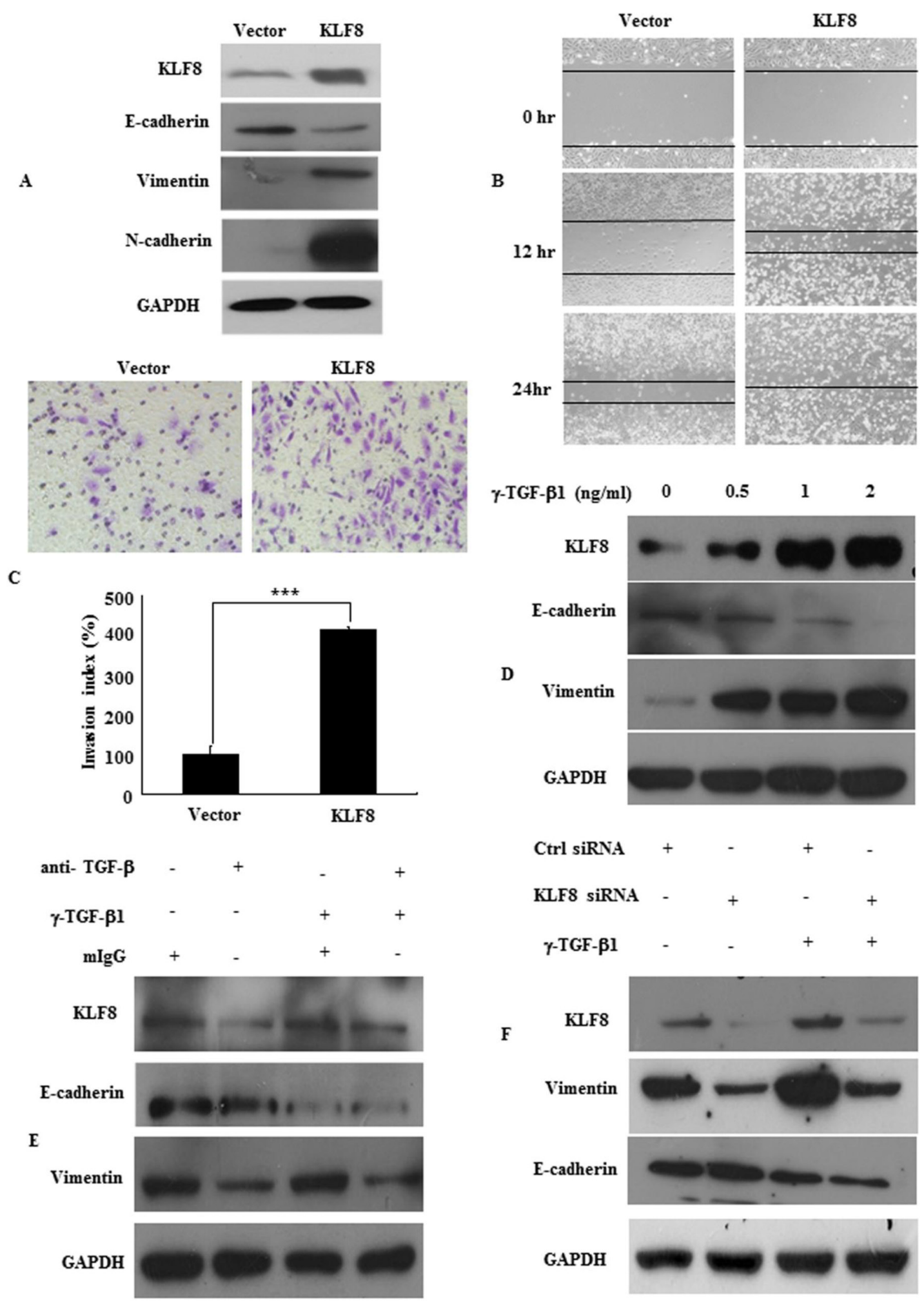

Figure 1: KLF8 upregulation promotes EMT. A. KLF8, E-cadherin, vimentin, N-cadherin expression in stable transfectants in LoVo cells, detected by western blot. B. Images of the wound closure of monolayer LoVo stable transfectants. Original magnification, 10×. C. Invasive potential of LoVo stable transfectants transfected with the vector, ${ }^{* *}, p<0.001$. D. KLF8 expression in LoVo cells treated with the indicated concentrations of recombinant $\gamma$-TGF- $\beta 1$ for $48 \mathrm{~h}$. The protein levels of KLF8, E-cadherin and vimentin were detected by western blot. E. LoVo cells were treated with recombinant TGF- $\beta 1(\gamma$-TGF- $\beta 1 ; 2 \mathrm{ng} / \mathrm{ml})$ in the presence of neutralizing anti-TGF- $\beta 1$ antibody $(\alpha-\mathrm{TGF}-\beta, 2 \mu \mathrm{g} / \mathrm{ml})$ or mouse IgG (mIgG) for $48 \mathrm{~h}$. The expression of KLF8, E-cadherin and vimentin was detected by western blot. F. Stable KLF8 transfectants were seeded in 6-well plates overnight and treated with $\gamma$-TGF- $\beta 1(2 \mathrm{ng} / \mathrm{ml})$ for an additional $48 \mathrm{~h}$. The expression of KLF8, E-cadherin and vimentin was detected by western blot. All figures are representative of four independent experiments with similar findings. 


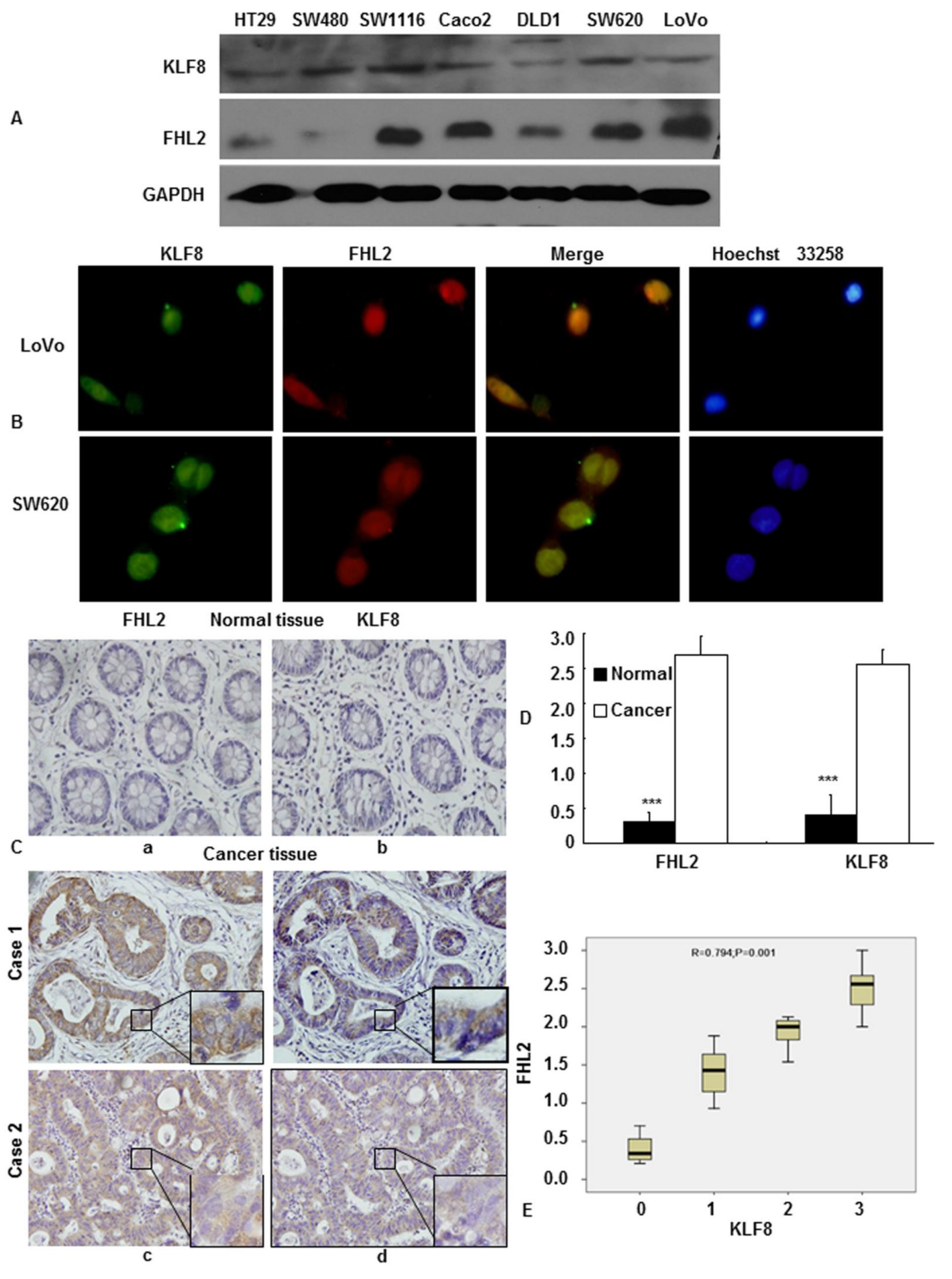

Figure 2: Expression profiles of KLF8 and FHL2 in colon cancer. A. KLF8 and FHL2 expression was detected in colon cancer cell lines by western blot. GAPDH was used as the internal control. B. Double staining of KLF8 and FHL2 in LoVo cells by an indirect immunofluorescence, with the nuclei counterstained by Hoechst 33258 (original magnification, 400×). C. FHL2 (a, c) and KLF8 (b, d) expression in normal or cancerous colon tissue specimen was detected by immunohistochemistry. These figures are representative of the patients. D. Average scores of the two proteins in normal and cancerous colon tissues. ${ }^{* *}, p<0.001$ between normal and cancer tissues. E. Positive staining for KLF8 and FHL2 was quantified, and their correlation was analyzed using Spearman's correlation method. Scale bars, $20 \mu \mathrm{m}$ in $\mathrm{B}$ and $100 \mu \mathrm{m}$ in $\mathrm{C}$. 
distributed in both the nucleus and cytoplasm of the same cancer cells (Figure 2C).

Semiquantitative scoring of the two proteins showed that the expression of both proteins in cancerous tissues was significantly higher than that of adjacent normal colon tissues (Figure 2D), and Spearman's correlation analysis showed a positive correlation between KLF8 and FHL2 expression (correlation coefficient $r=0.794, p<0.01$, Figure 2E).

\section{FHL2 is a direct target for transcriptional activation by KLF8}

We next assessed whether KLF8 proteins could directly bind to the FHL2 promoter. We scanned the promoter region $(<500 \mathrm{bp})$ of human FHL2 for the GTbox consensus sequence and found three potential binding sites: -50 to -55 (GT-box 1), -196 to -201 (GT-box 2) and -493 to -498 (GT-box 3 ) from the transcription initiation site (Figure 3A). To determine whether KLF8 binds to the FHL2 promoter region in vivo, we performed ChIP analysis using an anti-KLF8 antibody in LoVo cells. PCR amplification (Figure 3B) showed that a band of $195 \mathrm{bp}$, containing the first possible binding ( -55 to -50$)$ site, was immunoprecipitated. Normal rabbit IgG was used as the negative control. No bands were evident in the immunoprecipitates for the other two possible binding sites (GT-box 2 and/or GT-box 3) or for the control IgG.

We then cloned the promoter regions GT-box 1, GT-box 2 and GT-box 3 of human FHL2 upstream of a luciferase gene in a reporter plasmid. Transient transfections were performed to investigate whether the FHL2 promoter containing only GT-box 1, the only GTbox 1 belonging to the FHL 2 promoter, was activated by KLF8 overexpression. The luciferase activity increased by $2.83 \pm 0.16$ fold compared with that of the vector $(p<$ $0.001)$. However, the reporters that contained GT-box 2 and/or GT-box 3 of FHL2 showed slightly higher or lower luciferase activity compared with that of the vectors ( $p$ $>0.05$; Figure $3 \mathrm{C}$ ). We next mutated two nucleotides of the identified GT-box 1 binding region (Figure 3D). These mutations greatly reduced the effect of KLF8 on FHL2 promoter activity (Figure 3E). These results suggested that the proximal GT-box at -55 is the main KLF8-binding site in the FHL2 promoter (Figure 3).

\section{KLF8 promotes FHL2-mediated cell proliferation and morphology in CRC}

We previously showed that knocking down KLF8 inhibited CRC cell proliferation (Shi X, submitted). To investigate whether the mechanism of KLF8 regulating FHL2 plays a role in cell proliferation, we downregulated FHL2 in KLF8-overexpressing cells using siRNA and confirmed this effect by western blot (Figure 4A). FHL2 downregulation decreased KLF8-mediated proliferation of
LoVo cells, as shown using a WST-1 assay. The OD 450 s of KLF8 + FHL2 siRNA cells were $0.2178 \% \pm 0.009 \%$, $0.2646 \% \pm 0.009 \%, 0.3703 \pm 0.01 \%$ and $0.5504 \% \pm$ $0.027 \%$ after culturing for $0,24,48$ and $72 \mathrm{~h}$, respectively, whereas those of KLF8 + src siRNA cells were $0.2122 \%$ $\pm 0.005 \%, 0.3148 \% \pm 0.012 \%, 0.6544 \% \pm 0.05 \%$ and $1.0136 \% \pm 0.05 \%$, respectively. A significant difference was found between the stable KLF8 + FHL2 siRNA and $\mathrm{KLF} 8+\operatorname{src}$ siRNA transfectants at 48 and $72 \mathrm{~h}(p<0.01$; Figure 4B).

We then examined the morphologic features of these cells. The stable vector transfectants displayed a round or flat morphology with a short cytoplasmic process. However, the KLF8 transfectants exhibited a spindlelike, fibroblastic morphology, which is one of the main characteristics of EMT. Long or dendritic-like cytoplasmic processes were visible under a phase-contrast microscope. FHL2 knockdown in KLF8-overexpressing cells led to EMT reversion (Figure 4C).

To further characterize KLF8, we stained F-actin using phalloidin staining. Compared with the empty vector-expressing cells, the stable high expression of LoVo of KLF8 cell was present throughout the cytoplasm and at the rim zone of the protrusion. Moreover, filopodia and lamellipodia were identified as dynamic cellular features on the cell membrane surfaces that require actin polymerization and are involved in cancer cell invasion and metastasis (Figure 4D). FHL2 knockdown in KLF8overexpressing cells led to the mesenchymal to epithelial transition (MET) process, which is the reverse of the EMT process.

Our previous results showed that FHL2 induced tumor cell EMT and maintained the invasive potential of cancer cells [23]. However, the role of FHL2 in KLF8-induced EMT in colorectal cells remains elusive. SiRNA-mediated repression of FHL2 was performed in LoVo-KLF8 cells and resulted in decreased vimentin expression as well as in the conversion from mesenchymal marker (vimentin and N-cadherin) to epithelial marker (E-cadherin) expression compared with the control (KLF8src siRNA) cells (Figure 4E). These results might indicate that FHL2 and KLF8 regulate cell growth, migration and invasion.

\section{KLF8 is required for FHL2-mediated EMT and metastatic phenotypes}

Immunofluorescence staining of E-cadherin and vimentin, visualized by microscopy, confirmed the EMTassociated shift in marker expression (Figure 5A). Next, we performed a wound-healing assay. Knockdown of FHL2 in KLF8-overexpressing cells led to a decreased the migratory potential of KLF8-overexpressing cells in vitro (Figure 5B). Similarly, FHL2 downregulation in KLF8overexpressing cells led to a decreased invasion potential of KLF8-overexpressing cells by 34.5\% (Figure 5C). 
Transcription Start site

A FHL2 promoter

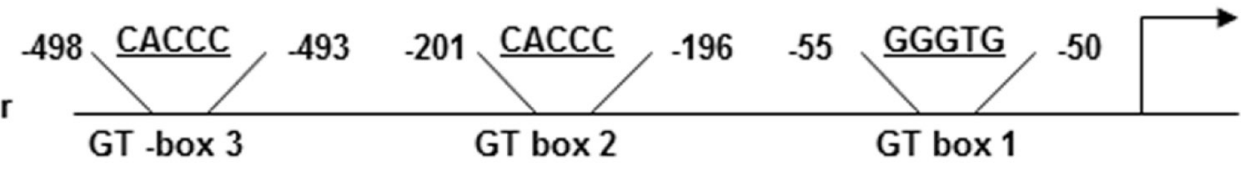

Input IgG KLF8-1 Input IgG KLF8-2 Input IgG KLF8-3

B

$300 \mathrm{bp}$

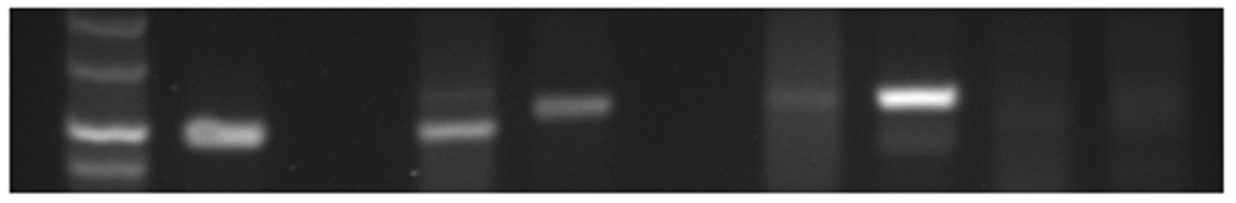

C

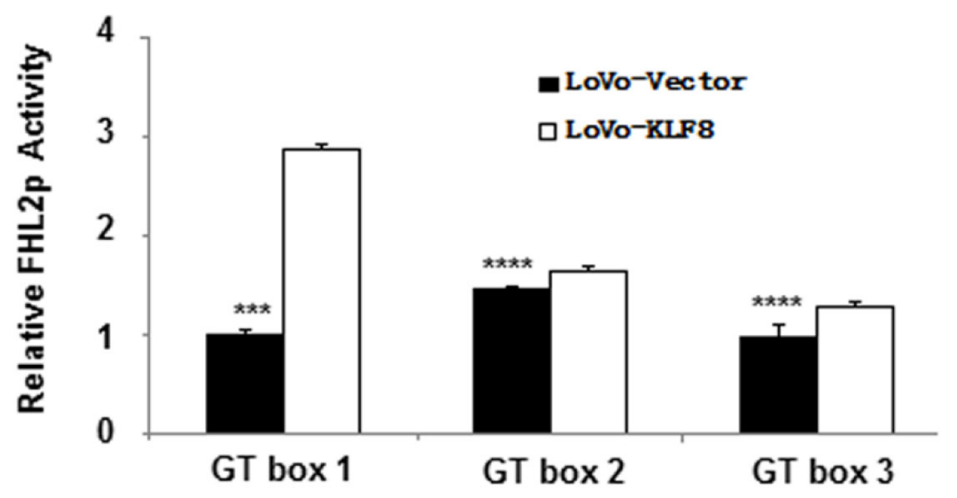

Wild type sequence of $\mathrm{GT}$ box 1

5'- GGGGGGTGCACAGAGGTGGAGC -3'

Mutated sequence of GT box 1

D

5'- GGGGAGTACACAGAGGTGGAGC -3'
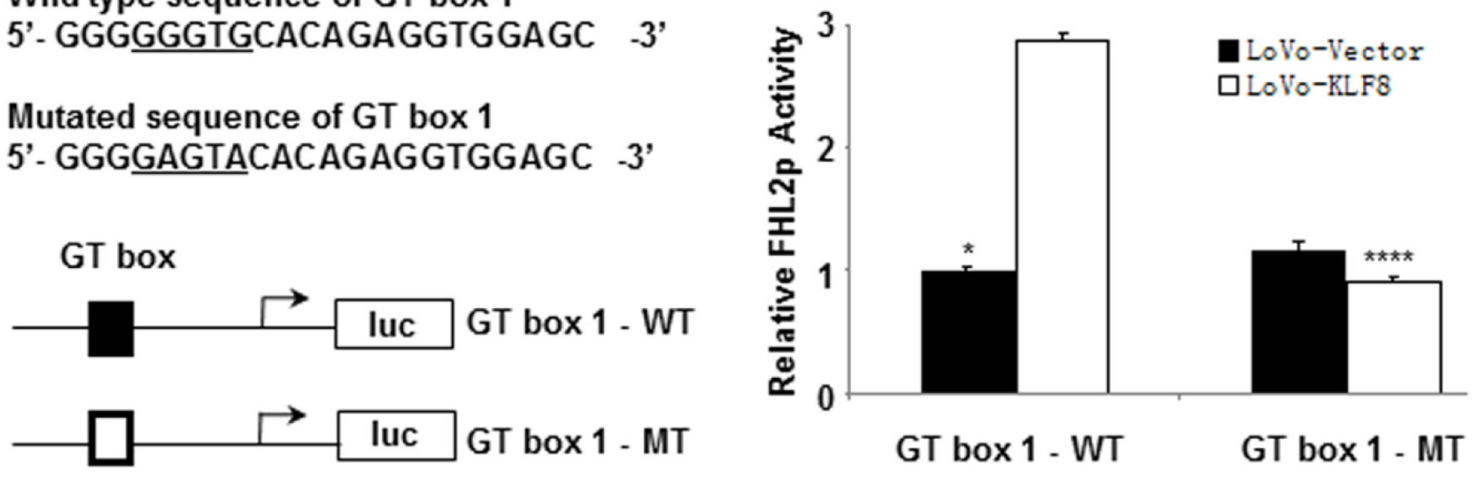

Figure 3: KLF8 directly binds to the promoter of FHL2. A. The luciferase (Luc) reporter constructs contained the FHL2 promoter with three potential KLF8 binding sites upstream of a luciferase gene. B. Chromatin immunoprecipitation (ChIP) was performed using an anti-KLF8 antibody or control IgG. The FHL2 promoter region to which KLF8 binds was significantly enriched after immunoprecipitation by an anti-KLF8 antibody. PCR products following ChIP were run on an ethidium-stained gel. C. KLF8 activates the FHL2 promoter (FHL2p) after co-transfection in LoVo cells. Luciferase activity was measured $48 \mathrm{~h}$ after transfection. Luciferase activity is expressed as the ratio of the promoter reporter activity to the control vector luciferase activity. RLU, relative luciferase units. $* * *, p<0.001 ; * * * *, p$ $>0.05$ D. Primer sequences used for site-directed mutations. A 136-bp (containing GT-box 1) FHL2 promoter fragment was cloned into pGL3 basic to generate pLuc55-wt. Site-directed mutagenesis of GT-box 1 in pLuc55 was performed to generate pLuc55-mt. The locations of GT-box 1 are labeled in bold, and the mutated nucleotides were underlined. wt, wildtype and mt, mutant. Diagram showing the GT-box

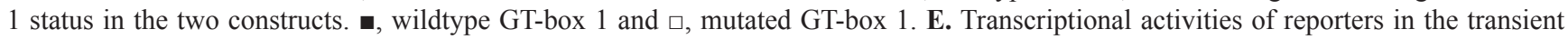
transfections. Dual luciferase assay were performed, and the results are expressed as fold induction of RLU. ***, $p<0.001 ; * * * *, p>0.05$. 
A

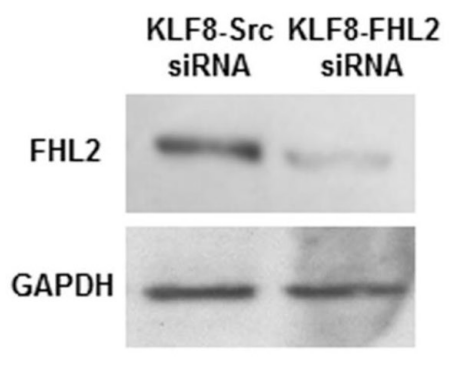

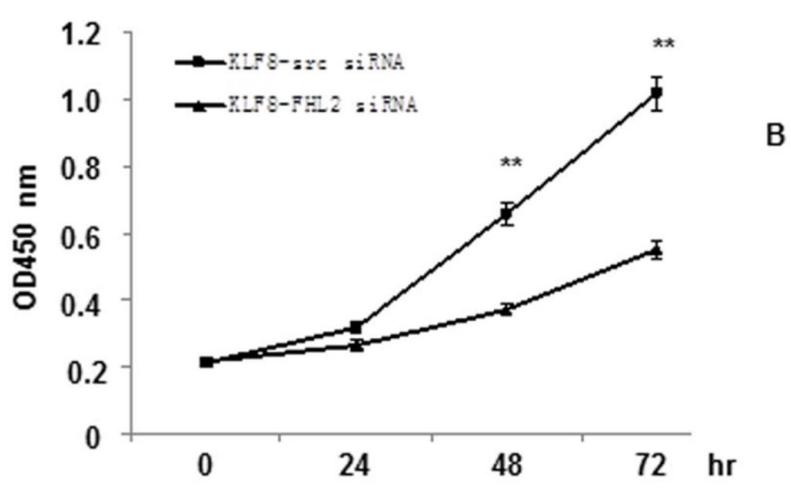

pcDNA3.1
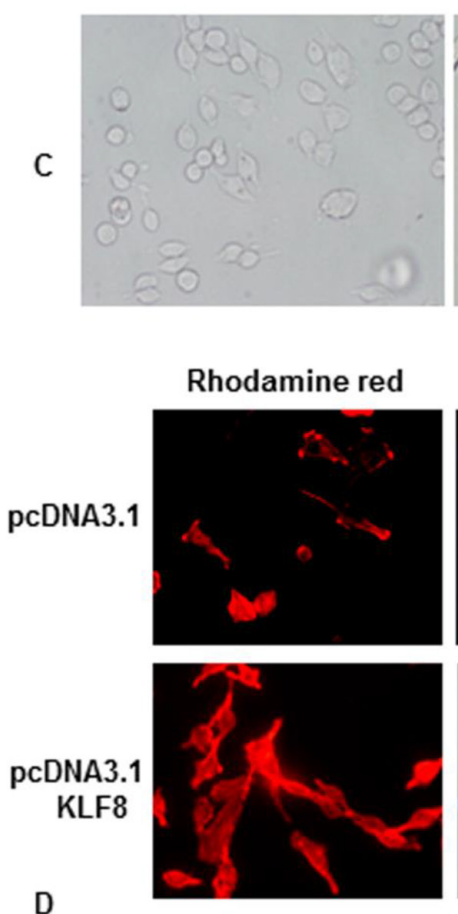

D
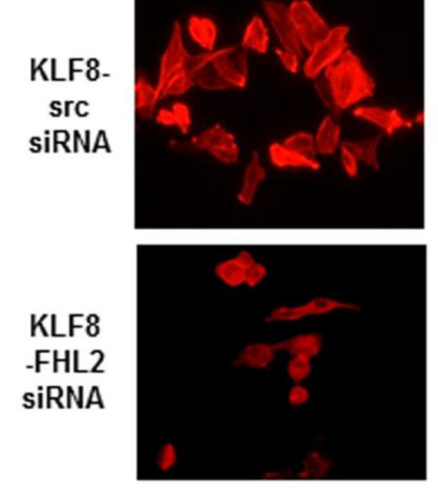

pcDNA3.1 -KLF8
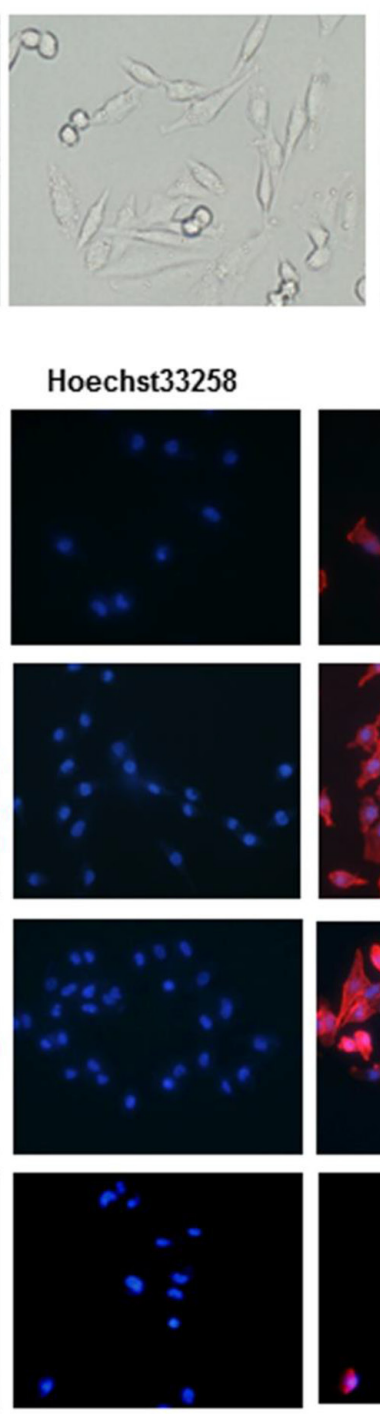

KLF8-src siRNA
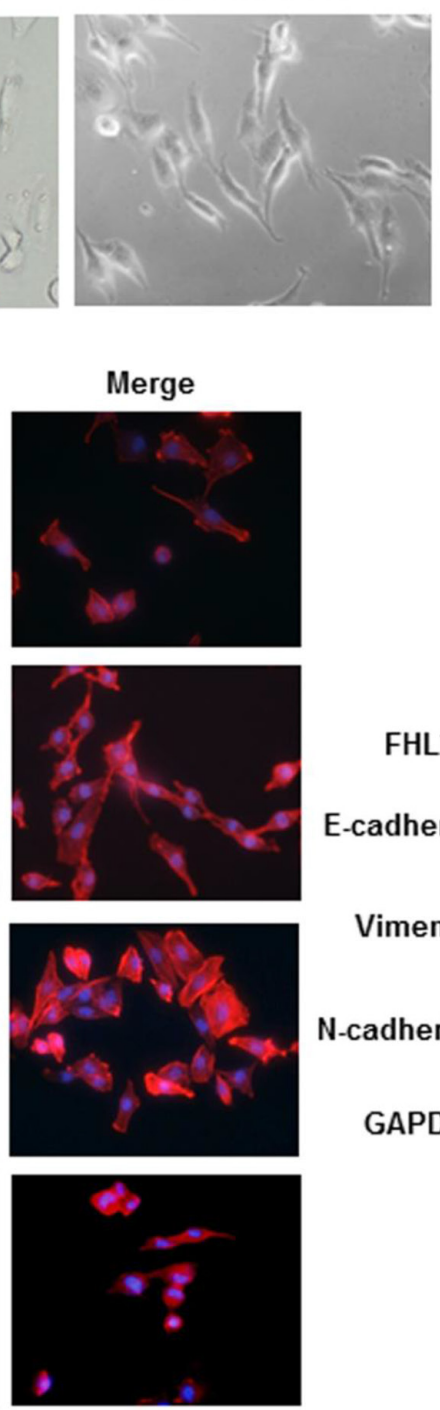

KLF8-FHL2-siRNA

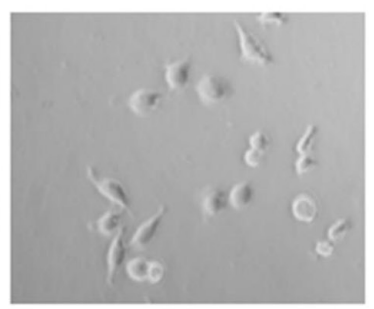

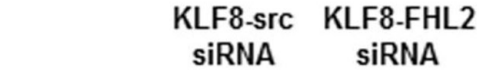

FHL2

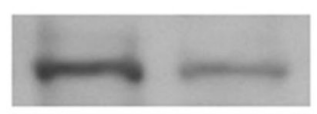

E-cadherin

\section{Vimentin}

N-cadherin

GAPDH
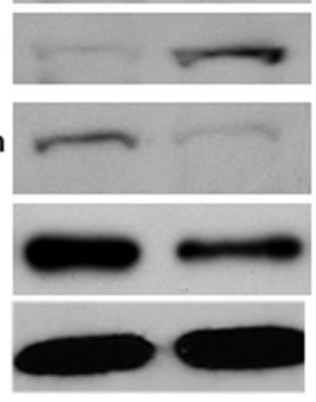

E

Figure 4: KLF8 promotes FHL2-mediated cell proliferation and morphology in CRC. A. Stable KLF8 transfectants were transfected with FHL2 siRNA, and FHL2 expression was detected by western blot $48 \mathrm{~h}$ later. B. LoVo/KLF8-src siRNA and LoVo/KLF8FHL2 siRNA cells seeded in 96-well tissue culture plates in triplicate and cultured in complete medium for 24,48 and $72 \mathrm{~h}$ were evaluated. Cell proliferation was assessed using the WST-1 assay. **, $p<0.01$. C. Morphology of LoVo/vector, LoVo/KLF8, LoVo/KLF8-src siRNA and LoVo/KLF8-FHL2 siRNA cells, visualized by phase-contrast microscopy. D. LoVo cells stained with rhodamine-phallotoxin for $48 \mathrm{~h}$ to identify F-actin filaments were visualized under fluorescent microscopy. All experiments were repeated 2 to 3 times with similar findings. E. EMT biomarkers, including E-cadherin, N-cadherin, vimentin, and FHL2, were detected by western blot $48 \mathrm{~h}$ after transfection. Scale bars represent $20 \mu \mathrm{m}$ in $\mathrm{C}$ and $\mathrm{D}$. 

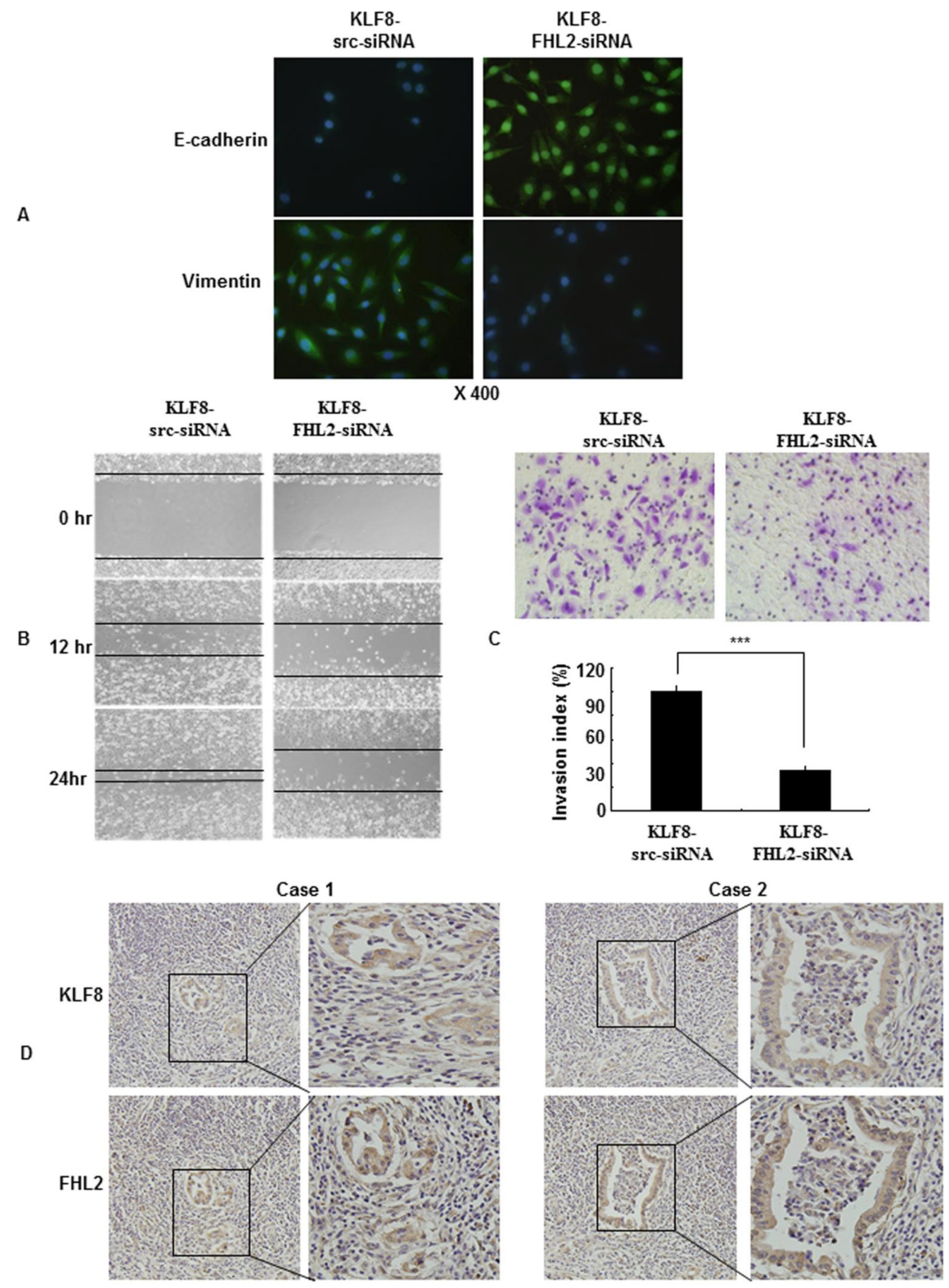

Figure 5: KLF8 is required for FHL2-mediated EMT and metastatic phenotypes. A. Immunofluorescence and microscopic visualization of E-cadherin (blue) and vimentin (green) staining in KLF8 src siRNA and KLF8-FHL2-siRNA cells. B. For the woundhealing experiments, cells were analyzed with live-cell microscopy. Original magnification, 10×. C. LoVo stable KLF8 transfectants were transfected with FHL2 siRNA $48 \mathrm{~h}$ later, and the invasive ability of the cells decreased. $* * *, p<0.001$. The experiments were repeated at least three times. D. Representative IHC images are shown for FHL2 and KLF8 expression in lymph node metastatic cancer tissues. Scale bars, $20 \mu \mathrm{m}$ in A and $100 \mu \mathrm{m}$ in $\mathrm{D}$. 


\section{Knockdown of FHL2 decreased the migratory and invasive potential of KLF8- overexpressing cells}

To validate our findings in vivo, we detected FHL2 and KLF8 in serial sections of lymph node metastatic cancer tissues as exemplified in two patients. We showed that FHL2 and KLF8 were expressed at high and intermediate levels, respectively, in the cytoplasm and nucleus of cancer cells (Figure 5D). This result confirmed the positive correlation between FHL2 and KLF8 by immunohistochemistry, which is consistent with previous reports.

Taken together, these results demonstrated the critical role of FHL2 in the induction of EMT and in the migration and invasion phenotypes caused by abnormal KLF8 expression.

\section{KLF8 promotes FHL2-mediated cell proliferation and metastasis in vivo}

To validate our findings in vivo, we inoculated vector, stable KLF8-transfectant and Lenti-KLF8-FHL2shRNA cells into BALB/c-nu/nu mice, as shown in Figure 6A. The tumor volumes of the KLF8-overexpressing cells were markedly greater than those of the vector-expressing cells. KLF8 overexpression progressed from a pronounced increase in vector cells at day 21 to a 4-fold increase in the cancer area 35 days after injection (Figure 6B). In contrast, tumors derived from FHL2 downregulation in KLF8overexpressing cells were markedly smaller than those of the vector-treated mice at 3 to 5 weeks (Figure 6B). FHL2 knockdown inhibited the proliferation of KLF8overexpressing LoVo CRC cells in vivo.

We next examined the expression of cell proliferation (Ki-67) and angiogenesis (CD105) markers at the protein levels in the xenograft tumors. Representative images of the tumors, stained by immunohistochemistry, are shown in Figure 6C. The KLF8 stable-transfectant group showed significantly increased proliferation rates and tumor vessel density than those in the vector group, whereas knockdown of FHL2 inhibited the growth rate and tumor vessel density in the KLF8-overexpressing group.

To investigate whether FHL2 is required for KLF8-induced metastatic phenotypes, shRNAmediated repression of FHL2 was performed in KLF8overexpression in LoVo after orthotopic implantation assays [35] (Figure 7A). The presence of CRC metastasis in the liver was confirmed by histological analysis (Figure 7B). The tumor volumes of the KLF8-overexpressing group were significantly increased compared with those of

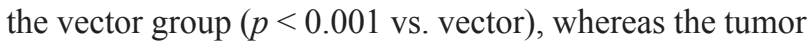
volumes after FHL2 knockdown were greatly decreased compared with those in the KLF8-overexpressing group on day 23 (Figure 7C). These results demonstrated that
FHL2 downregulation in KLF8-overexpressing cells inhibited CRC cell metastasis to the liver.

To further demonstrate whether KLF8 is required for FHL2-mediated EMT, repression of FHL2 in KLF8overexpressing cells was performed at the mRNA level in orthotopic xenograft tumors. KLF8 overexpression resulted in a significant loss of the epithelial marker E-cadherin, whereas FHL2 downregulation in KLF8overexpressing cells increased E-cadherin (Figure 7D). Thus, KLF8 is suggested to play a critical role in the induction of EMT via FHL2 expression.

Taken together, these results clearly indicated that the KLF8-FHL2 axis plays an important role in CRC development and metastasis.

\section{DISCUSSION}

In this report, we identified the KLF8-mediated regulation of FHL2 as a novel mechanism for the promotion of human CRC cell proliferation, invasion and metastasis. First, we showed a strong correlation between the expression of KLF8 and FHL2 in CRC cell lines. Second, we determined that KLF8 promoted FHL2 transcription and activity. Third, our data showed that KLF8 had a critical role in FHL2-mediated tumor growth, EMT and metastatic phenotypes in vitro and in vivo. These findings suggested that the cooperative relationship between FHL2 and KLF8 plays a pivotal role in CRC progression and metastasis.

KLF8 is a GT-box binding dual-transcription factor, and KLF8 overexpression has been noted in a variety of aggressive human carcinomas [16, 19]. Interestingly, several studies on KLF8 have indicated its involvement in the early steps of metastasis. For example, KLF8 was shown to stimulate the invasion of breast cancer cells through the induction of the matrix metalloproteinase gene MMP-9 [17]. Our results showed that KLF8 overexpression induced EMT and promoted migration and metastasis in CRC cells. These findings implied that aberrant upregulation of KLF8 might be an important mechanism underlying cancer metastasis.

TGF- $\beta 1$ has also been shown to promote tumor progression and metastasis in established cancers, in part through the induction of EMT [23, 36-38]. This apparent conversion in TGF- $\beta 1$ function after tumor initiation is known as the "TGF- $\beta 1$ paradox." It is important to understand the downstream effectors of the TGF- $\beta 1$ signaling pathway and to identify the "molecular switch" or "switches" mediating the "TGF- $\beta 1$ paradox." TGF- $\beta 1$ is considered a major driver of the EMT in tumors. While investigating the mechanistic interactions between TGF- $\beta 1$ and KLF8 signaling during induction of EMT in CRC, we found that induction of EMT in LoVo cells by TGF- $\beta 1$ was associated with a significant increase in KLF8 expression. When KLF8 expression was silenced, TGF- $\beta 1$ induction of the EMT phenotype was abrogated, 
A
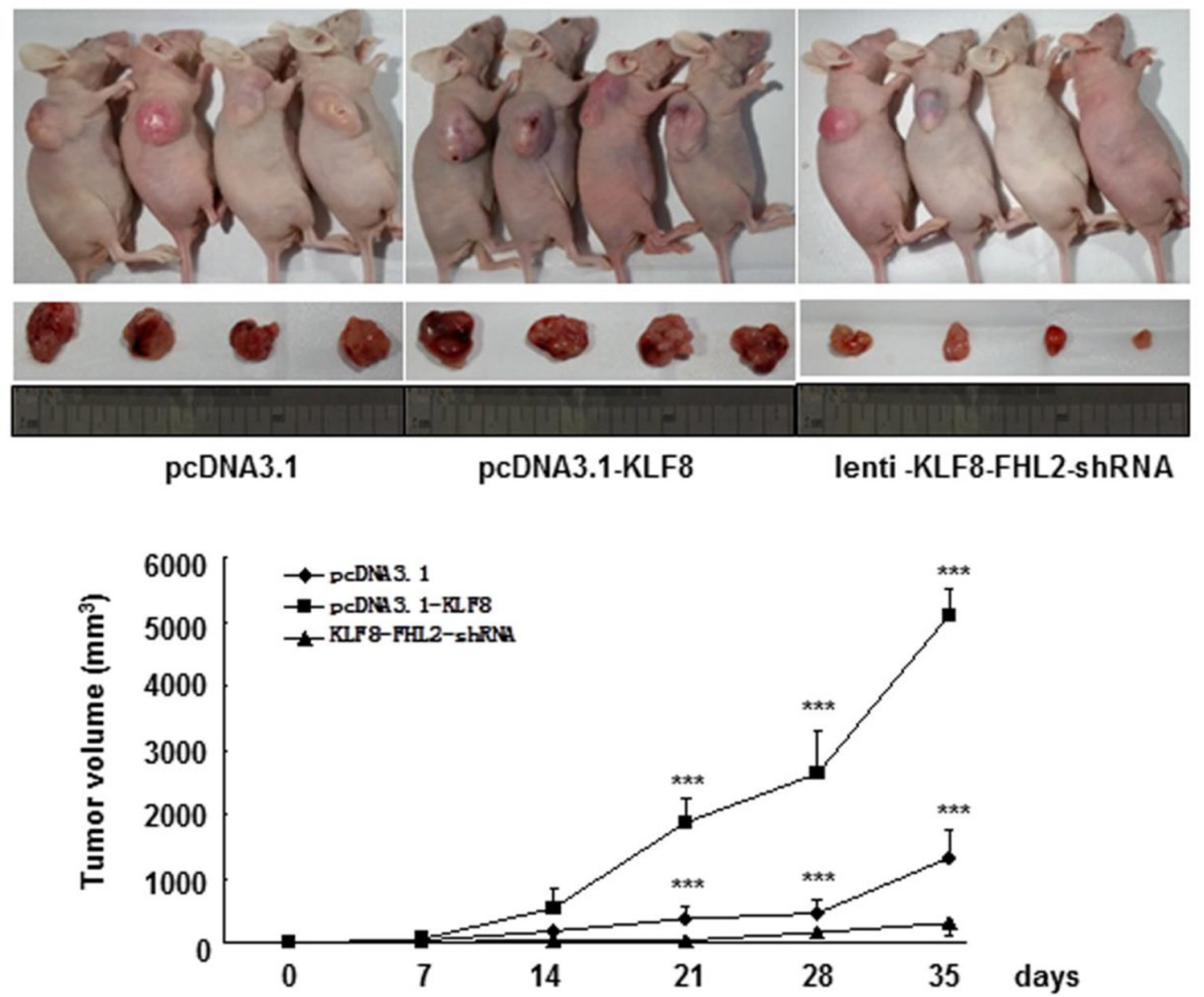

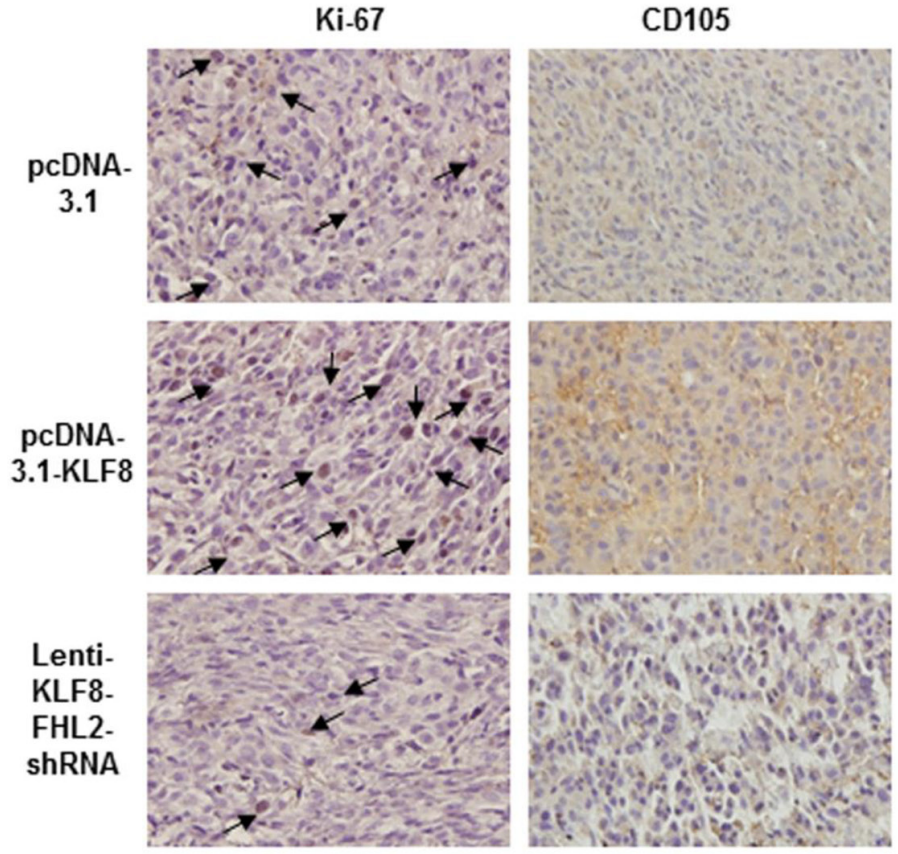

$\mathrm{C}$
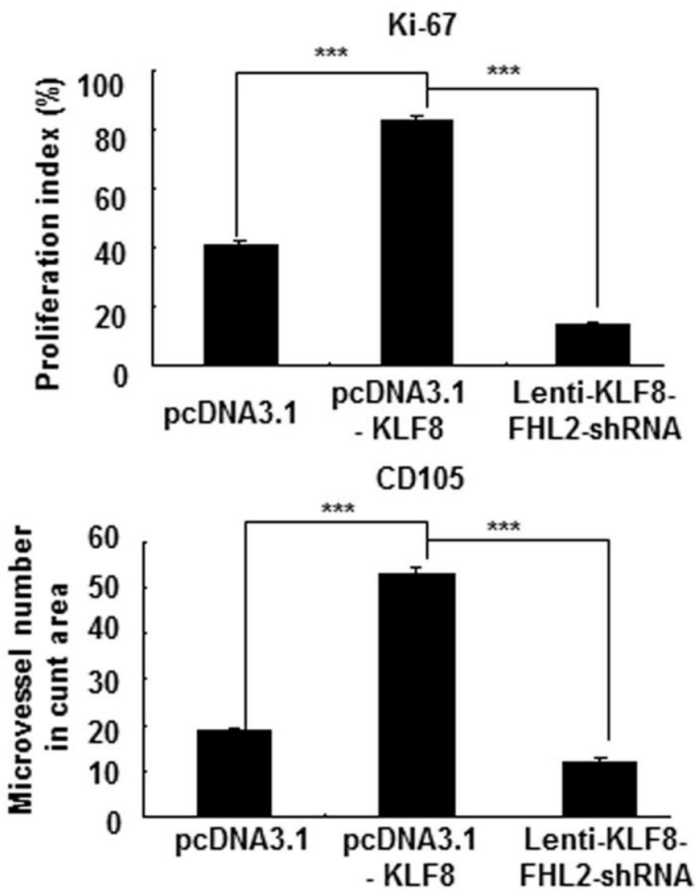

D

Figure 6: KLF8 promotes FHL2-mediated cell proliferation in CRC in vivo. A. LoVo cells $\left(5 \times 10^{7}\right)$ were injected subcutaneously in the right flanks of nude mice. One week after tumor cell injection, equal amounts of vector, KLF8 or KLF8-FHL2shRNA cells were directly injected into the tumors at three different positions. Images shown were captured on day 35 after injection. B. Tumor size was measured weekly after tumor cell inoculation in each group. ${ }^{* * *}, p<0.001$, vector vs. KLF8 and KLF8 vs. KLF8-FHL2shRNA, respectively. C. FHL2 knockdown significantly inhibited KLF8-induced proliferation (Ki-67, ***, $p<0.001$, vector vs. KLF8 and KLF8 or KLF8-FHL2-shRNA, respectively), and a considerable decrease of tumor vessel density (CD105, ***, $p<0.001$, vector vs. KLF8 and KLF8 or KLF8-FHL2-shRNA) was observed by immunohistochemistry. Scale bars represent $100 \mu \mathrm{m}$. 
A
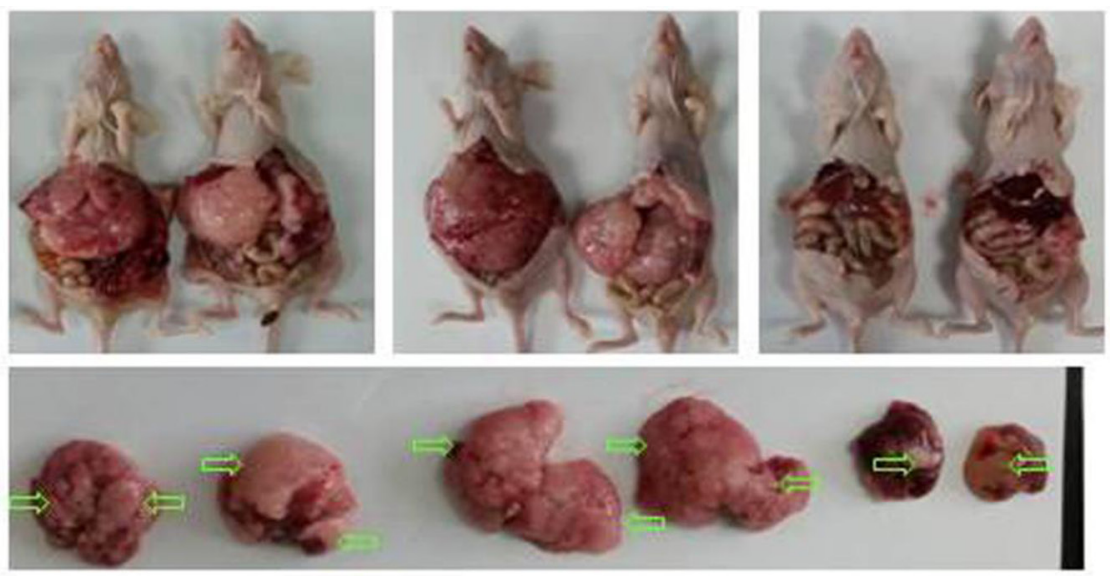

pcDNA3.1

pcDNA3.1-KLF8

Lenti-KLF8-
FHL2-shRNA

B

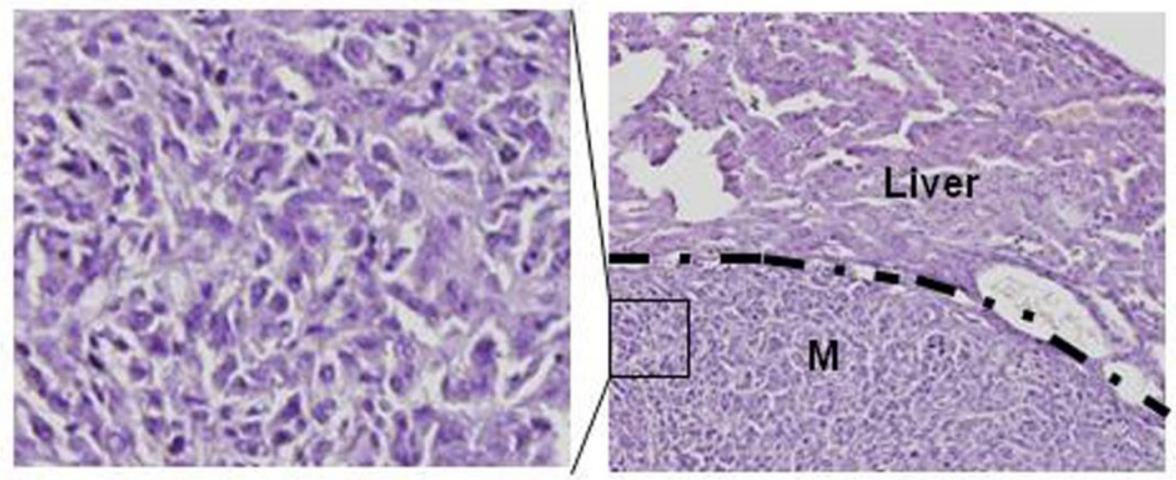

H\&E staining, $X 100$

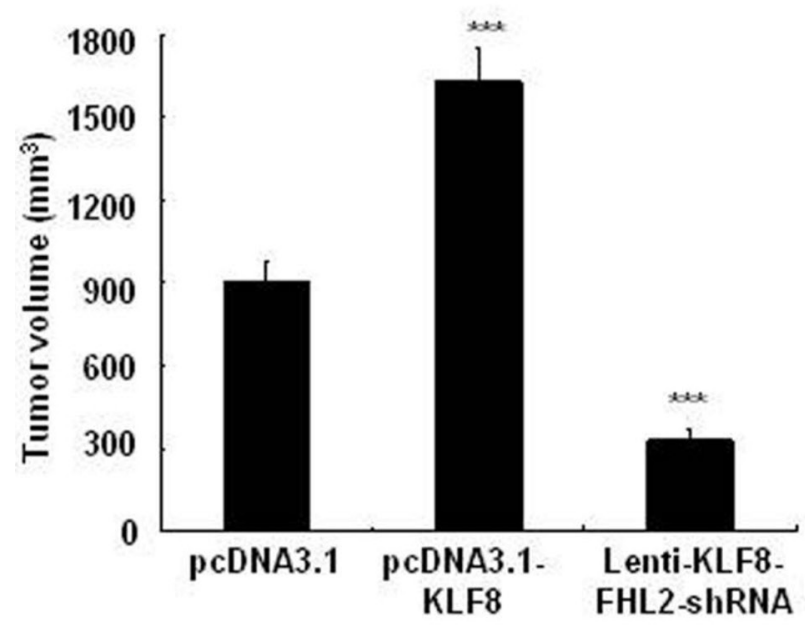

C

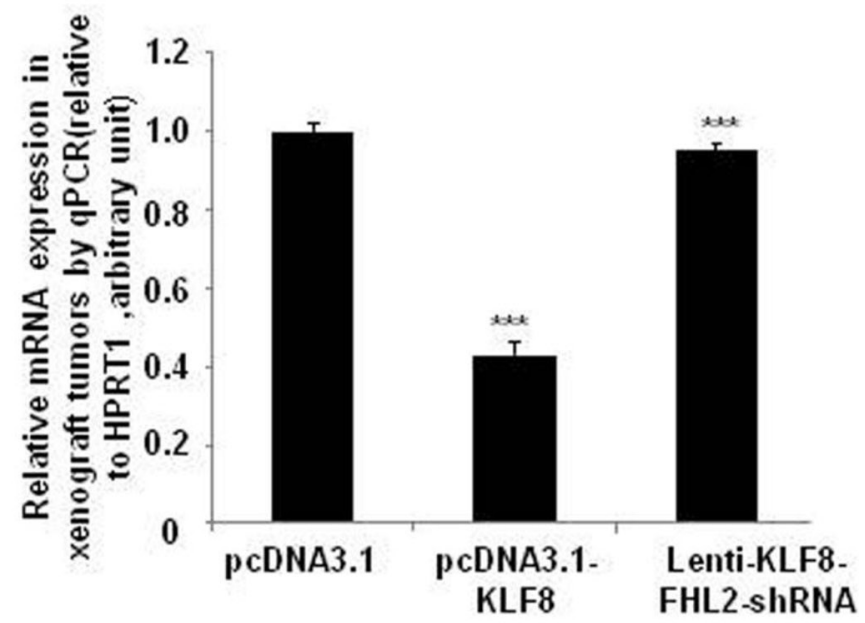

D

Figure 7: KLF8 is required for FHL2-mediated EMT and liver metastasis in orthotopic tumors after intrasplenic injection. A. Necropsy images of mice with orthotopically implanted CRC, with metastatic loci marked by arrows. B. The mice were sacrificed, and metastatic cancer tissues were stained with H\&E. Scale bars, $100 \mu \mathrm{m}$. C. Tumor volumes (mean \pm standard error; ***, $p<0.001$, vector vs. KLF8; KLF8 vs. KLF8-FHL2-siRNA) in mice measured on the last day of the experiment, at autopsy $(n=2)$. D. The expression of E-cadherin in tumors derived from LoVo cells was determined by qPCR. ***, $p<0.001$, vector vs. KLF8; KLF8 vs. KLF8FHL2-siRNA. 
and KLF8 expression was also decreased. We suggest that KLF8 might act as a potent inducer of EMT in CRC cells.

FHL2 is a member of the FHL protein family [20].

The role of FHL2 in cancer is particularly intriguing because FHL2 binds to different proteins [24-26]. In rhabdomyosarcoma cells, FHL2 overexpression induced apoptosis [20]. In melanoma cells, however, the synergistic overexpression of SKI and FHL2 enhanced cell growth [28]. Thus, it appears that FHL2 can regulate tumorigenesis in multiple human cancers [23, 27]. However, the roles of KLF8 and FHL2 in CRC are unknown. In this study, we showed that the distribution pattern of FHL2 was highly congruent with that of KLF8 and that FHL2 protein expression was highly correlated with KLF8 expression by immunohistochemistry and immunofluorescence. Moreover, FHL2 knockdown resulted in a marked blockage of KLF8 expression in vitro. Therefore, we speculated that KLF8 and FHL2 might play roles in CRC progression.

The $\mathrm{Sp} 1 / \mathrm{KLF}$ family members are related in that they bind GC-box and CACCC-box sequences in gene promoter regions [17]. We previously demonstrated that Sp1 stimulated FHL2 expression in GI cancer cells through interaction with its binding element located at the promoter of the FHL2 gene [3]. However, whether FHL2 is involved with KLF8 function remains unclear. In the present study, we identified that FHL2 is a direct transcriptional target of KLF8, evidenced by KLF8 directly binding to and activating the FHL2 promoter. This result provides a mechanism by which KLF8 induced FHL2-mediated EMT and potentially contributed to CRC growth and metastasis. The conclusion is based on the following observations. First, we showed that KLF8 directly binds to the FHL2 promoter by ChIP and luciferase reporter assays. The results from these studies identified the sequence from -55 to $-50 \mathrm{bp}$ as GT-box 1; furthermore, mutation of GT-box 1 resulted in blockage of KLF8 transcriptional activity. Second, we further investigated whether KLF8 contributed to FHL2-induced EMT. By silencing FHL2 expression in LoVo cells transfected with a KLF8-expressing plasmid, we found that the EMT phenotype was reversed. Third, FHL2 knockdown inhibited the EMT and metastatic potential induced by KLF8 overexpression in CRC in vivo. Together, these results strongly suggested that the FHL2-induced EMT and metastasis were mediated through KLF8.

In summary, we identified FHL2 as a novel target for transcriptional activation by KLF8 and demonstrated that the regulation of FHL2 by KLF8 is critical for human $\mathrm{CRC}$ cell growth and invasion. We have also shown that KLF8 expression was important for human CRC EMT and metastasis and played an important role in CRC cell progression and aggression. Therefore, KLF8 might represent a novel favorable target for intervention against CRC cell proliferation and metastasis.

\section{MATERIALS AND METHODS}

\section{Reagents and antibodies}

Recombinant human TGF- $\beta 1$ (240-B) and monoclonal anti-TGF- $\beta 1$ antibody were purchased from R\&D Systems (Minneapolis, MN, USA). Mouse antihuman FHL2, used for western blotting, was purchased from MBL International Incorporation (Woburn, Japan). Rabbit anti-human KLF8 antibody, used for western blotting and IHC, was purchased from Aviva Systems Biology (San Diego, CA, USA). Prediluted rabbit antihuman vimentin, N-cadherin and E-cadherin, KLF8 and mouse anti-human glyceraldehyde-3- phosphate dehydrogenase (GAPDH) antibodies, used for western blotting and/or immunohistochemistry, were purchased from Abcam (Cambridge, UK). Bovine anti-mouse IgGTR and goat anti-rabbit IgG-FITC antibodies purchased from Santa Cruz (Santa Cruz, CA, USA). Goat antirabbit immunoglobulins/HRP, rabbit anti-mouse immunoglobulins/HRP, and normal mouse IgG antibodies were purchased from Dako (Carpinteria, CA, USA).

The human colon cancer cell lines HT29, SW480, SW1116, Caco2, DLD1, SW620 and LoVo were obtained from American Type Culture Collection (ATCC, Rockville, MD, USA) and cultured as previously described [16].

\section{Immunohistochemistry of human CRC cancer samples}

The Ethics Committee of the Southern Medical University of China approved our experimental protocols. Thirteen pairs of surgically resected colon cancer tissues and adjacent normal tissues were collected from both primary and metastatic (lymph node) cancer sites (Department of Pathology, Nanfang Hospital, First Affiliated Hospital of Southern Medical University, China). The expression of FHL2 and KLF8 was visualized as described previously. The tissues in which more than $10 \%$ of cancer cells were positively stained were considered positive. For the quantitative analysis, the ratio of positively stained cells to all of the tumor cells in five random areas at 200 -fold magnification was recorded. Scoring of the tissue slides was independently performed by two investigators; the percentage of positive cells and the intensity of staining were scored from 0 to 3 as follows: 0 , less than $10 \%$ of cells stained; $1,10-50 \%$ of cells stained; $2,50-75 \%$ of cells stained; and 3 , more than $75 \%$ of cells stained.

\section{Constructs and establishment of stable transfectants}

The KLF8 open reading frame and 3'-untranslated region (774 bp) were cloned into pcDNA3.1(+) in a previous study [19]. To establish stable cell lines, cells 
transfected with empty pcDNA 3.1 vector and pcDNA3.1KLF8 were passaged 1:15 (vol/vol) and cultured in RPMI 1640 medium (Invitrogen, Carlsbad, CA) supplemented with $800 \mu \mathrm{g} / \mathrm{ml}$ geneticin (G418, Calbiochem, Darmstadt, Germany Canada) for 4 weeks.

\section{Cell migration and invasion assays}

Cell migration was assessed using a wound-healing assay. Cells plated in six-well plates at $90-95 \%$ confluence were wounded with a pipette tip at time 0 . The speed of wound closure was monitored, and the cells were imaged at different time points using a phase-contrast microscope. The media were changed to remove cell debris, and the cells were cultured in the presence of $10 \mu \mathrm{g} / \mathrm{ml}$ mitomycin $\mathrm{C}$ to inhibit cell proliferation. Images were captured $24 \mathrm{~h}$ later.

Cell invasion was assessed using a Matrigel invasion chamber (BD Biosciences), following the protocol provided by the manufacturer. After incubation for 48 hours, the noninvasive cells were removed with a cotton swab. Invasive cells were stained with $0.2 \%$ crystal violet and counted under a microscope. The average number of cells in five fields per membrane was counted in triplicate inserts. The invasion index was expressed as the percentage of test cells to that of treatments cells or control.

\section{siRNA transfection}

The siRNA duplexes consisted of 21 base pairs with a 2-base deoxynucleotide overhang (Proligo, Singapore). The siRNA sequences were as follows: KLF8 sense strand, ((NM_007250), 5'-CGAUAUGGAUAAACUCAUAdTdT-3'; and FHL2 sense strand, (NM_201557), 5'-CGAAU CUCUCUUUG GCAAGdTdT-3'. The scrambled (src) siRNA (5'-TTC TCCGAACGTGTCACGT-3'), which does not target any gene, was used as the negative control. The siRNA transfection was performed using Lipofectamine 2000 (Invitrogen, USA), according to the manufacturer's instructions.

\section{Construction and transfection of lentiviral vectors}

To further investigate the effects of siRNA-induced down-regulation of FHL2 in KLF8-overexpressed cells on tumor growth in vivo, an FHL2-RNAi lentiviral vector (pGCSIL-FHL2shRNA) was constructed (Shanghai GeneChem Co, Ltd, Shanghai, China) [19]. Doublestranded oligonucleotides encoding human FHL2-vshRNA (CCGGCCGAATCTCTCTTTGGCAAGTCAAGAGCT TGCCAAAGAGAGATTCGTTTTTG) were inserted into the short hairpin RNA (shRNA) expression vector pGCSIL (Shanghai GeneChem Co., Ltd.), and the identities of the clones were verified by sequencing.
A recombinant lentiviral vector was produced by co-transfecting HEK293T cells with the lentiviral expression vector and the packaging plasmid mix using Lipofectamine 2000, according to the manufacturer's instructions.

The titer of the purified virus was determined in 293 T cells using the serial dilution method.

For lentiviral transduction, LoVo cells were subcultured at $1 \times 10^{5}$ cells/well in 6-well culture plates and were then transduced with FHL2-siRNAexpressing (FHL2 siRNA) or src-siRNA-expressing lentivirus at a 50 multiplicity of infection. The cells were harvested $72 \mathrm{~h}$ after infection, and the transduction efficiency was evaluated by counting the percentage of GFP-positive cells.

\section{Western blot assays}

For western blot analysis, cells were harvested and lysed in lysis buffer. A total of $30 \mu \mathrm{g}$ of protein lysates were separated by SDS-PAGE and transferred onto a PVDF membrane. Primary antibodies were diluted according to the company's recommendation. Proteins were visualized using the enhanced chemiluminescence detection system.

\section{Reverse transcription PCR (RT-PCR) and quantitative real-time reverse transcription PCR (qRT-PCR)}

Total RNA extraction and reverse transcription were performed as previously described [31]. Quantitative realtime RT-PCR was performed using Applied Biosystems Sequence Detection System 7900 (ABI Prism 7900HT, Applied Biosystems Company, Foster City) with $10 \mathrm{ml}$ of a mixture comprising Power SYBR GREEN PCR Master Mix Applied Biosystems Company, $500 \mathrm{nmol}$ of each primer and $300 \mathrm{ng}$ of complementary DNA template. The reactions were performed with an initial denaturation step of $95^{\circ} \mathrm{C}$ for $5 \mathrm{~min}$, followed by 60 cycles of $20 \mathrm{~s}$ at $94^{\circ} \mathrm{C}, 20$ $\mathrm{s}$ at $55^{\circ} \mathrm{C}$ and $40 \mathrm{~s}$ at $72^{\circ} \mathrm{C}$. A final extension at $72^{\circ} \mathrm{C}$ for 5 min was included before a temperature ramp from $72^{\circ} \mathrm{C}$ to $95^{\circ} \mathrm{C}$ at $0.1^{\circ} \mathrm{C} / \mathrm{s}$ with continuous fluorescent acquisition. Each complementary DNA sample was run in duplicate for each real-time PCR assay, and the average relative fold messenger RNA levels were determined using the $2^{-\Delta \Delta \mathrm{Ct}}$ method, with HPRT1 detected as the internal control $[39,40]$.

\section{Promoter reporter and dual luciferase assays}

First, 55-bp, 201-bp and 498-bp fragments of the FHL2 promoter upstream of the transcription start site were cloned into the pGL3basic vector. The pLuc55 mutant construct was created by site-directed mutagenesis of the FHL2 promoter vector (Stratagene, La Jolla, CA, USA). The primer sequences are listed in Supplementary Table S1. 
For the luciferase assay, the cells were transiently transfected with the various pLuc constructs with Lipofectamine 2000, as previously reported [23]. The firefly and Renilla luciferase activities were measured using the Dual-Luciferase reporter system (Promega) with a model TD-20/20 Luminometer (EG\&G, Berthold, Australia). The firefly luciferase activity value was normalized to the Renilla activity value. The transcriptional activity at the promoter was presented as the fold induction of relative luciferase units (RLUs) compared with the basic pGL3 vector control. The RLU was the value of the firefly luciferase unit divided by the value of the Renilla luciferase unit.

\section{Chromatin immunoprecipitation (ChIP) assays}

ChIP assays were performed according to the protocol provided by the ChIP assay kit (Upstate Cell Signaling Solutions, Lake Placid, NY, USA). The lysates were incubated either with antibodies specific for KLF8 or with normal mouse IgG. The PCR reaction generated 195bp, 225-bp and 228-bp products from the FHL2 proximal promoter containing GT-box 1 ( -56 to $-51 \mathrm{bp})$, GT-box 2 ( -202 to $-197 \mathrm{bp}$ ) and GT-box 3 ( -499 to $-494 \mathrm{bp}$ ), respectively. The primers and antibodies used in the ChIP assays are listed in Supplementary Table S1.

\section{Immunofluorescence}

Cells grown on glass coverslips were fixed with $4 \%$ paraformaldehyde, and non-specific binding was blocked. The coverslips were probed with primary antibodies, followed by Texas red (TR)- or FITC-conjugated secondary antibodies. The nuclei were counterstained with $1 \mu \mathrm{g} / \mathrm{ml}$ Hoechst 33258. After mounting, the coverslips were visualized under a Zeiss Axioskop fluorescence microscope.

\section{WST-1 cell proliferation assay}

Scr-siRNA and KLF8-siRNA cells were seeded into 96-well plates at $0.5-1 \times 10^{4}$ cells/well and cultured in RPMI 1640 for 24, 48 and $72 \mathrm{~h}$. Then, $10 \mu \mathrm{l}$ of the WST1 reagent (Roche Diagnostics, Mannheim, Germany) was added to each well, and the plates were incubated for $4 \mathrm{~h}$ at $37^{\circ} \mathrm{C}$. The amount of formazan was quantified using an ELISA reader at an absorbance of $450 \mathrm{~nm}$.

\section{In vivo tumor growth assay}

Tumor growth was evaluated in a nude mouse xenograft model. LoVo cells $\left(5 \times 10^{6}\right)$, in $0.1 \mathrm{ml}$ of RPMI, were inoculated subcutaneously into the right flanks of 5-6-week-old female BALB/c-nu/nu mice (Laboratory Animal Unit, Southern Medical University, China), and the resulting tumor sizes were measured weekly. Institutional guidelines were followed for the handling of the animals.
The mice were maintained under sterile conditions. The tumor volumes were calculated as follows: total tumor volume $\left(\mathrm{mm}^{3}\right)=\mathrm{L} \times \mathrm{W}^{2} / 2$, where $\mathrm{L}$ is the length, and $\mathrm{W}$ is the width. On day 35 after inoculation, the mice were sacrificed, and the tumors were dissected and weighed. Immunohistochemistry analysis was performed using antiKi-67, MMP-9 and anti-CD105 antibodies.

\section{In vivo metastasis assays}

The mice were anesthetized with isoflurane. For the orthotopic tumor implantation assays, pcDNA3.1-, pcDNA3.1-KLF8- or lenti-KLF8-FHL2-siRNA-expressing cells $\left(1 \times 10^{6}\right.$ in $0.1 \mathrm{ml}$ of PBS $)$ were inoculated into the dorsal subcostal incision to expose the spleen. The volume $(50 \mu \mathrm{L})$ of tumor cell suspension was slowly injected into the spleen using a 25-gauge needle. After 23 days, the mice were euthanized. The weight and volume of the livers were measured for each mouse. Metastatic tissues were microscopically examined with H\&E staining.

\section{Statistical analyses}

The results obtained from duplicated q-RT-PCR assays and triplicated luciferase experiments were expressed as the mean $\pm \mathrm{SD}$. The significance of the patient specimen data was determined using the Pearson correlation coefficient. The significance of the in vitro and in vivo data was determined using Student's $t$ test (twotailed). $P$ values less than 0.05 were considered significant.

\section{ACKNOWLEDGMENTS AND FUNDING}

This study was supported by grants from the National Natural Science Funds of China (81172057 \& 81272761), 'President Foundation of Nanfang Hospital, Southern Medical University' (2012B009, 2013Z007), and high-level topic-matching funds of Nanfang Hospital (201347 and G201227). Guangdong Provincial Key Laboratory of Gastroenterology, Department of Gastroenterology, Nanfang Hospital, Southern Medical University; Guangzhou Pilot Project of Clinical and Translational Research Center (early gastrointestinal cancer, No.7415696196402).

\section{CONFLICTS OF INTEREST}

The authors declare no conflict of interest.

\section{REFERENCES}

1. Jackson SP, MacDonald JJ, Lees-Miller S, Tjian R. GC box binding induces phosphorylation of $\mathrm{Sp} 1$ by a DNAdependent protein kinase. Cell. 1990; 63:155-65.

2. Chen X, Johns DC, Geiman DE, Marban E, Dang DT, Hamlin G, Sun R, Yang VW. Krüppel-like factor 4 
(gut-enriched Krüppel-like factor) inhibits cell proliferation by blocking G1/S progression of the cell cycle. J Biol Chem. 2001; 276:30423-8.

3. Guo Z, Zhang W, Xia G, Niu L, Zhang Y, Wang X, Zhang Y, Jiang B, Wang J. Sp1 upregulates the four and half $\lim 2$ (FHL2) expression in gastrointestinal cancers through transcription regulation. Mol Carcinog. 2010; 49:826-36.

4. Hong JS, Kim SW, Koo JS. Sp1 up-regulates cAMPresponse- element- binding protein expression during retinoic acid-induced mucous differentiation of normal human bronchial epithelial cells. Biochem J. 2008; 410:49-61.

5. Abdelrahim M, Smith R 3rd, Burghardt R, Safe S. Role of Sp proteins in regulation of vascular endothelial growth factor expression andproliferation of pancreatic cancer cells. Cancer Res. 2004; 64:6740-9.

6. Ai W, Liu Y, Langlois M, Wang TC. Kruppel-like factor 4 (KLF4) represses histidine decarboxylase gene expression through an upstream $\mathrm{Sp} 1$ site and downstream gastrin responsive elements. J Biol Chem. 2004; 279:8684-93.

7. Yori JL, Johnson E, Zhou G, Jain MK, Keri RA. Kruppellike factor 4 inhibits epithelial-to-mesenchymal transition through regulation of E-cadherin gene expression. J Biol Chem. 2010; 285:16854-63.

8. Godmann M, Gashaw I, Katz JP, Nagy A, Kaestner KH, Behr R. Krüppel-like factor 4, a "pluripotency transcription factor" highly expressed in male postmeiotic germ cells, is dispensable for spermatogenesis in the mouse. Mech Dev. 2009; 126:650-64.

9. Reeves HL, Narla G, Ogunbiyi O, Haq AI, Katz A, Benzeno S, Hod E, Harpaz N, Goldberg S, Tal-Kremer S, Eng FJ, Arthur MJ, Martignetti JA, Friedman SL. Kruppellike factor 6 (KLF6) is a tumor-suppressor gene frequently inactivated in colorectal cancer. Gastroenterology. 2004; 126:1090-103.

10. Spörl F, Korge S, Jürchott K, Wunderskirchner $M$, Schellenberg K, Heins S, Specht A, Stoll C, Klemz R, Maier B, Wenck H, Schrader A, Kunz D, Blatt T, Kramer A. Krüppel-like factor 9 is a circadian transcription factor in human epidermis that controls proliferation of keratinocytes. Proc Natl Acad Sci U S A. 2012; 109:10903-8.

11. Guillaumond F, Gréchez-Cassiau A, Subramaniam M, Brangolo S, Peteri-Brünback B, Staels B, Fiévet C, Spelsberg TC, Delaunay F, Teboul M. Kruppel-like factor KLF10 is a link between the circadian clock and metabolism in liver. Mol Cell Biol. 2010; 30:3059-70.

12. Buck A, Buchholz M, Wagner M, Adler G, Gress T, Ellenrieder V. The tumor suppressor KLF11 mediates a novel mechanism in transforming growth factor betainduced growth inhibition that is inactivated in pancreatic cancer. Mol Cancer Res. 2006; 4:861-72.

13. Gumireddy K, Li A, Gimotty PA, Klein-Szanto AJ, Showe LC, Katsaros D, Coukos G, Zhang L, Huang Q. KLF17 is a negative regulator of epithelial-mesenchymal transition and metastasis in breast cancer. Nat Cell Biol. 2009; 11:1297-304.

14. Nandan MO, Yoon HS, Zhao W, Ouko LA, Chanchevalap S, Yang VW. Krüppel-like factor 5 mediates the transforming activity of oncogenic H-Ras. Oncogene. 2004; 23:3404-13.

15. Nakamura Y, Migita T, Hosoda F, Okada N, Gotoh M, Arai Y, Fukushima M, Ohki M, Miyata S, Takeuchi K, Imoto I, Katai H, Yamaguchi T, Inazawa J, Hirohashi S, Ishikawa Y, Shibata T. Krüppel-like factor 12 plays a significant role in poorly differentiated gastric cancer progression. Int J Cancer. 2009; 125:1859-67.

16. Wang X, Zheng M, Liu G, Xia W, McKeown-Longo PJ, Hung MC, Zhao J. Krüppel-like factor 8 induces epithelial to mesenchymal transition and epithelial cell invasion. Cancer Res. 2007; 67:7184-93.

17. Wang $\mathrm{X}, \mathrm{Lu} \mathrm{H}$, Urvalek AM, Li T, Yu L, Lamar J, DiPersio CM, Feustel PJ, Zhao J. KLF8 promotes human breast cancer cell invasion and metastasis by transcriptional activation of MMP9. Oncogene. 2011; 30:1901-11.

18. Lu H, Wang X, Urvalek AM, Li T, Xie H, Yu L, Zhao J. Transformation of human ovarian surface epithelial cells by Krüppel-like factor 8. Oncogene. 2014; 33:10-8.

19. Li JC, Yang XR, Sun HX, Xu Y, Zhou J, Qiu SJ, Ke AW, Cui YH, Wang ZJ, Wang WM, Liu KD, Fan J. Up-regulation of Krüppel-like factor 8 promotes tumor invasion and indicates poor prognosis for hepatocellular carcinoma. Gastroenterology. 2010; 139:2146-2157.

20. Genini M, Schwalbe P, Scholl FA, Remppis A, Mattei MG, Schäfer BW. Subtractive cloning and characterization of DRAL, a novel LIM-domain protein down-regulated in rhabdomyosarcoma. DNA Cell Biol. 1997; 16:433-42.

21. Zhang W, Yang Y, Jiang B, Peng J, Tu S, Sardet C, Zhang Y, Pang R, Hung IF, Tan VP, Lam CS, Wang J, Wong BC. XIAP-associated factor 1 interacts with and attenuates the trans-activity of four and a Half LIM protein 2. Mol Carcinog. 2011; 50:199-207.

22. Lin J, Lin S, Yu X, Choy PC, Shen X, Deng C, Kuang S, $\mathrm{Wu}$ J. The four and a half LIM domain protein 2 interacts with and regulates the HERG channel. FEBS J. 2008; 275:4531-9.

23. Zhang W, Jiang B, Guo Z, Sardet C, Zou B, Lam CS, Li J, He M, Lan HY, Pang R, Hung IF, Tan VP, Wang J, Wong BC. Four-and-a-half LIM protein 2 promotes invasive potential and epithelial-mesenchymal transition in colon cancer. Carcinogenesis. 2010; 31:1220-9.

24. Morlon A, Sassone-Corsi P. The LIM-only protein FHL2 is a serum-inducible transcriptional coactivator of AP-1. Proc Natl Acad Sci U S A. 2003; 100:3977-82.

25. Fimia GM, De Cesare D, Sassone-Corsi P. A family of LIM-only transcriptional coactivators: tissue-specific expression and selective activation of CREB and CREM. Mol Cell Biol. 2000; 20:8613-22. 
26. Purcell NH, Darwis D, Bueno OF, Müller JM, Schüle R, Molkentin JD. Extracellular signal-regulated kinase 2 interacts with and is negatively regulated by the LIM-only protein FHL2 in cardiomyocytes. Mol Cell Biol. 2004; 24:1081-95.

27. Morgan MJ, Whawell SA. The structure of the human LIM protein ACT gene and its expression in tumor cell lines. Biochem Biophys Res Commun. 2000; 273:776-83.

28. Chen D, Xu W, Bales E, Colmenares C, Conacci-Sorrell M, Ishii S, Stavnezer E, Campisi J, Fisher DE, Ben-Ze'ev A, Medrano EE. SKI activates Wnt/beta-catenin signaling in human melanoma.Cancer Res. 2003; 63:6626-34.

29. Li M, Wang J, Ng SS, Chan CY, Chen AC, Xia HP, Yew DT, Wong BC, Chen Z, Kung HF, Lin MC. The Fourand-a-half-LIM Protein 2 (FHL2) Is Overexpressed in Gliomas and Associated with Oncogenic Activities. Glia. 2008; 56:1328-38.

30. Kahl P, Gullotti L, Heukamp LC, Wolf S, Friedrichs N, Vorreuther R, Solleder G, Bastian PJ, Ellinger J, Metzger E, Schüle R, Buettner R. Androgen receptor coactivators lysine-specific histone demethylase 1 and four and a half LIM domain protein 2 predict risk of prostate cancer recurrence. Cancer Res. 2006; 66:11341-7.

31. Zhang W, Wang J, Zou B, Sardet C, Li J, Lam CS, Ng L, Pang R, Hung IF, Tan VP, Jiang B, Wong BC. Four and a half LIM protein 2 (FHL2) negatively regulates the transcription of E-cadherin through interaction with Snail1. Eur J Cancer. 2011; 47:121-30.

32. Zhou BP, Deng J, Xia W, Xu J, Li YM, Gunduz M, Hung MC. Dual regulation of Snail by GSK-3beta-mediated phosphorylation in control of epithelial-mesenchymal transition. Nat Cell Biol. 2004; 6:931-40.
33. Yang $\mathrm{MH}, \mathrm{Wu} \mathrm{MZ}$, Chiou SH, Chen PM, Chang SY, Liu CJ, Teng SC, Wu KJ. Direct regulation of TWIST by HIF-1alpha promotes metastasis. Nat Cell Biol. 2008; 10:295-305.

34. Yang WH, Lan $\mathrm{HY}$, Huang $\mathrm{CH}$, Tai SK, Tzeng $\mathrm{CH}$, Kao SY, Wu KJ, Hung MC, Yang MH. RAC1 activation mediates Twist1-induced cancer cell migration. Nat Cell Biol. 2012; 14:366-74.

35. Wang S, Zhou J, Wang XY, Hao JM, Chen JZ, Zhang XM, Jin H, Liu L, Zhang YF, Liu J, Ding YQ, Li JM. Downregulated expression of SATB2 is associated with metastasis and poor prognosis in colorectal cancer. J Pathol. 2009; 219:114-22.

36. Morrison CD, Parvani JG, Schiemann WP. The relevance of the TGF- $\beta$ Paradox to EMT-MET programs. Cancer Lett. $2013 ; 341: 30-40$.

37. Wendt MK, Tian M, Schiemann WP. Deconstructing the mechanisms and consequences of TGF- $\beta$-induced EMT during cancerprogression. Cell Tissue Res. 2012; 347:85-101.

38. Han G, Lu SL, Li AG, He W, Corless CL, Kulesz-Martin M, Wang XJ. Distinct mechanisms of TGF-beta1-mediated epithelial-to-mesenchymal transition and metastasis during skin carcinogenesis. J Clin Invest. 2005; 115:1714-23.

39. Hellemans J, Vandesompele J. Selection of reliable reference genes for RT-qPCR analysis. Methods Mol Biol. 2014; 1160:19-26.

40. de Kok JB, Roelofs RW, Giesendorf BA, Pennings JL, Waas ET, Feuth T, Swinkels DW, Span PN. Normalization of gene expression measurements in tumor tissues: comparison of 13 endogenous control genes. Lab Invest. 2005; 85:154-9. 